Emma D'Aniello, Dipartimento di Matematica e Applicazioni R. Caccioppoli, Università degli Studi di Napoli "Federico II," Via Cintia, Complesso Monte S. Angelo, 80126 Napoli, Italy. e-mail: daniel@matna2.dma.unina.it

\title{
ON THE EXISTENCE OF VECTOR MEASURES WITH GIVEN MARGINALS
}

\begin{abstract}
A type of Strassen's Theorem for measures taking values in the positive cone of a Banach lattice is proved. We generalize a result of A. Hirshberg and R. M. Shortt and formulate a type of Strassen's Theorem in a topological context via closed sets.
\end{abstract}

\section{Introduction}

In joint work, M. März and R. M. Shortt [10, Theorem 3.7] generalize a version of the theorem known in probability theory as "Strassen's Theorem" (see [13], $[6],[5, \S 11.6])$ to the context of measures assuming values in a reflexive Banach lattice.

Continuing the line of inquiry of [12], in [8, Theorem 2] A. Hirshberg and R. M. Shortt prove a result of this type for measures taking values in Banach lattices of a certain type: the KB-spaces. Since reflexive $\Rightarrow \mathrm{KB} \Rightarrow$ order complete, their result is a generalization of [10, Theorem 3.7].

In this paper we give a formulation of Strassen's Theorem for measures taking values in Banach lattices with order continuous norm [Theorem 3.10]. These spaces occupy a position between the KB-spaces and the order complete Banach lattices (reflexive $\Rightarrow \mathrm{KB} \Rightarrow$ order continuous norm $\Rightarrow$ order complete), hence our result is a generalization of [8, Theorem 2].

We also formulate a type of Strassen's Theorem in a topological context via closed sets [Theorem 3.15].

\footnotetext{
Key Words: Banach lattice, vector measure

Mathematical Reviews subject classification: Primary 28B05; Secondary 46B42

Received by the editors October 30, 1998

* This research has been partially supported by Ministero dell'Università e della Ricerca Scientifica e Tecnologica (Italy) and Programma di Ricerca e di Interesse Nazionale Analisi Reale.
} 


\section{Preliminaries}

Let $\mathcal{F}$ be a field of subsets of a set $X$. Let $\mathbf{B}$ be a Banach space and $\mathbf{B}^{*}$ its dual.

We remind that a vector measure $\mu: \mathcal{F} \rightarrow \mathbf{B}$ is an additive set function, i.e., $\mu\left(F_{1} \cup F_{2}\right)=\mu\left(F_{1}\right)+\mu\left(F_{2}\right)$, for disjoint $F_{1}$ and $F_{2}$ in $\mathcal{F}$. We denote by $c a(\mathcal{F}, \mathbf{B})$ the vector space of all countably additive vector measures $\mu: \mathcal{F} \rightarrow \mathbf{B}$ and by $\|\mu\|$ the semivariation of $\mu[4]$.

A class $\mathcal{K}$ of subsets of a set $X$ is compact if it has the following property: given a sequence $\left(K_{n}\right)_{n \in N}$ drawn from $\mathcal{K}$ such that $K_{1} \cap \ldots \cap K_{n} \neq \emptyset$ for each $n \in N$, the intersection $\cap_{n=1}^{\infty} K_{n}$ is non-empty. Let $\mathcal{F}$ be a field of subsets of $X$ and let $\mu: \mathcal{F} \rightarrow \mathbf{B}$ be a vector measure taking values in a Banach space B. We say that $\mu$ is a compact measure if there exists a compact class $\mathcal{K}$ of subsets of $X$ such that, for every $F \in \mathcal{F}$ and $\epsilon>0$, there are sets $F^{\prime} \in \mathcal{F}$ and $K \in \mathcal{K}$ with $F^{\prime} \subseteq K \subseteq F$ and $\|\mu\|\left(F-F^{\prime}\right)<\epsilon$. In this case we say that the class $\mathcal{K} \mu$-approximates $\mathcal{F}$.

Now suppose that $\mathcal{F}$ is a $\sigma$-field. We say that a vector measure $\mu: \mathcal{F} \rightarrow \mathbf{B}$ is perfect if the restriction of $\mu$ to every countably generated sub- $\sigma$-field of $\mathcal{F}$ is compact.

Given a Hausdorff topological space $X$ and its Borel $\sigma$-field $\mathcal{B}(X)$, a vector measure $\mu: \mathcal{B}(X) \rightarrow \mathbf{B}$ is tight if, for each $\epsilon>0$ and set $B \in \mathcal{B}(X)$, there is some compact set $K \subseteq B$ such that $\|\mu\|(B-K)<\epsilon$. Clearly, every tight measure on the Borel $\sigma$-field of a metric space is compact.

A Banach lattice $\mathbf{B}$ is a $K B$-space if every increasing norm bounded sequence of its positive cone $\mathbf{B}^{+}$is norm convergent [1, Definition 14.10].

A normed vector lattice $\mathbf{B}$ is said to have order continuous norm [11, Definition 5.12] if every order convergent filter in $\mathbf{B}$ norm converges. For information on these spaces, see $([1],[11])$. In these sources are to be found the following results.

1. A countably order complete Banach lattice $\mathbf{B}$ has order continuous norm iff no Banach sublattice of $\mathbf{B}$ is vector lattice isomorphic to $l^{\infty}[11$, Theorem 5.14].

2. Every KB-space has order continuous norm [11, page 92]. The converse is not true: the vector lattice $c_{0}$ of real null sequences under the supremum norm is an important example of a Banach lattice with order continuous norm, but it is not KB.

3. Every Banach lattice having order continuous norm is order complete [11, page 92]. The converse is not true: $l^{\infty}$ is order complete, but it has 
not order continuous norm.

\section{Strassen's Theorem}

Without further notice, in this section, for two sets $X_{1}$ and $X_{2}$,

1. $\mathcal{P}\left(X_{1} \times X_{2}\right)$ is the power set of $X_{1} \times X_{2}$,

2. for every $F \in \mathcal{P}\left(X_{1} \times X_{2}\right), \chi_{F}$ is the indicator function of $F$,

3. $\pi_{1}: X_{1} \times X_{2} \rightarrow X_{1}$ and $\pi_{2}: X_{1} \times X_{2} \rightarrow X_{2}$ are the projections of the product space $X_{1} \times X_{2}$,

4. if $X_{1}$ and $X_{2}$ are Hausdorff topological spaces, by $\mathcal{B}\left(X_{1}\right), \mathcal{B}\left(X_{2}\right)$ and $\mathcal{B}\left(X_{1} \times X_{2}\right)$ we denote the Borel $\sigma$-fields of $X_{1}, X_{2}$ and $X_{1} \times X_{2}$, respectively.

Definition 3.1. Let $X_{1}$ and $X_{2}$ be Hausdorff topological spaces and let $\mu_{1}$ and $\mu_{2}$ be tight elements of $c a\left(\mathcal{B}\left(X_{1}\right), \mathbf{B}^{+}\right)$and of $c a\left(\mathcal{B}\left(X_{2}\right), \mathbf{B}^{+}\right)$, respectively, such that $\mu_{1}\left(X_{1}\right)=\mu_{2}\left(X_{2}\right)=\alpha$, where $\mathbf{B}^{+}$is the positive cone of an order complete Banach lattice $\mathbf{B}$. Let $M=\left\{\mu \in c a\left(\mathcal{B}\left(X_{1} \times X_{2}\right), \mathbf{B}^{+}\right)\right.$: $\mu$ is tight and $\mu \circ \pi_{1}^{-1}=\mu_{1}$ and $\left.\mu \circ \pi_{2}^{-1}=\mu_{2}\right\}$ (i.e. $M=\left\{\mu \in \operatorname{ca}\left(\mathcal{B}\left(X_{1} \times\right.\right.\right.$ $\left.\left.X_{2}\right), \mathbf{B}^{+}\right): \mu$ is tight and has marginals $\mu_{1}$ and $\left.\left.\mu_{2}\right\}\right)$. For every $F \in \mathcal{P}\left(X_{1} \times\right.$ $X_{2}$ ) we define

$$
S(F)= \begin{cases}0 & \text { if } M=\emptyset \\ \bigvee\left\{\mu^{*}(F): \mu \in M\right\} & \text { otherwise }\end{cases}
$$

where 0 is the zero element of $\mathbf{B}$ and $\mu^{*}(F)$ is the outer measure of $F$,

$$
\begin{gathered}
\text { and } \\
I(F)=\bigwedge\left\{\sum_{i=1}^{2} \mu_{i}\left(B_{i}\right): B_{i} \in \mathcal{B}\left(X_{i}\right) \text { and } F \subseteq \cup_{i=1}^{2} \pi_{i}^{-1}\left(B_{i}\right)\right\} .
\end{gathered}
$$

For the properties of $I$ and $S$ see [3] and [9].

Theorem 3.2. Let $C \in \mathcal{B}\left(X_{1} \times X_{2}\right)$ be a closed set. Then

$$
I(C)=\bigwedge\left\{\sum_{i=1}^{2} \mu_{i}\left(C_{i}\right): C_{i} \text { closed set in } \mathcal{B}\left(X_{i}\right) \text { and } C \subseteq \cup_{i=1}^{2} \pi_{i}{ }^{-1}\left(C_{i}\right)\right\} \text {. }
$$


Proof. The proof consists of four steps.

1. First we shall prove that, for every $F \in \mathcal{B}\left(X_{1} \times X_{2}\right)$,

$(*) \quad I(F)=\bigwedge\left\{\sum_{i=1}^{2} \int f_{i} d \mu_{i}: f_{i} \in \mathcal{L}\left(X_{i}\right)\right.$ and $\left.\chi_{F} \leq \sum_{i=1}^{2} f_{i} \circ \pi_{i}\right\}$,

where $\mathcal{L}\left(X_{1}\right)$ is the family of all $\mu_{1}$-integrable Borel measurable functions defined on $X_{1}$, with values in $[0,1]$, and $\mathcal{L}\left(X_{2}\right)$ is the family of all $\mu_{2}$-integrable Borel measurable functions defined on $X_{2}$, with values in $[0,1]$.

Notice that if $B_{i} \in \mathcal{B}\left(X_{i}\right)$ with $F \subseteq \cup_{i=1}^{2} \pi_{i}{ }^{-1}\left(B_{i}\right)$ then $\chi_{F} \leq \sum_{i=1}^{2} \chi_{B_{i}} \circ \pi_{i}$, where $\chi_{B_{i}}$ is the indicator function of $B_{i}$.

Let $f_{1}$ and $f_{2}$ be any two elements of $\mathcal{L}\left(X_{1}\right)$ and of $\mathcal{L}\left(X_{2}\right)$, respectively, satisfying $\chi_{F} \leq \sum_{i=1}^{2} f_{i} \circ \pi_{i}$. Then this inequality leads to the relation

$F \subseteq\left(\left\{y \in X_{1}: f_{1}(y) \geq s\right\} \times X_{2}\right) \cup\left(X_{1} \times\left\{z \in X_{2}: f_{2}(z) \geq 1-s\right\}\right)$ for $0 \leq s \leq 1$.

Let $x^{*}$ be a nonnegative element of $\mathbf{B}^{*}$. Then, since $x^{*} \circ \mu_{1}$ and $x^{*} \circ \mu_{2}$ are real-valued measures, as in Proposition 3.3 of [9],

$$
\begin{aligned}
& \sum_{i=1}^{2} \int f_{i} d\left(x^{*} \circ \mu_{i}\right)=\int_{0}^{1}\left(x^{*} \circ \mu_{1}\right)\left(\left\{y \in X_{1}: f_{1}(y) \geq s\right\}\right) d s+ \\
& \int_{0}^{1}\left(x^{*} \circ \mu_{2}\right)\left(\left\{z \in X_{2}: f_{2}(z) \geq 1-s\right\}\right) d s \\
& \geq \inf _{0 \leq s \leq 1} x^{*}\left(\mu_{1}\left(\left\{y \in X_{1}: f_{1}(y) \geq s\right\}\right)+\mu_{2}\left(\left\{z \in X_{2}: f_{2}(z) \geq 1-s\right\}\right)\right) .
\end{aligned}
$$

Therefore, for every nonnegative element $x^{*}$ of $\mathbf{B}^{*}$,

$$
\begin{aligned}
& x^{*}\left(\sum_{i=1}^{2} \int f_{i} d \mu_{i}\right)=\sum_{i=1}^{2} \int f_{i} d\left(x^{*} \circ \mu_{i}\right) \\
& \geq \inf _{0 \leq s \leq 1} x^{*}\left(\mu_{1}\left(\left\{y \in X_{1}: f_{1}(y) \geq s\right\}\right)+\mu_{2}\left(\left\{z \in X_{2}: f_{2}(z) \geq 1-s\right\}\right)\right) \\
& \geq x^{*}\left(\bigwedge_{0 \leq s \leq 1}\left(\mu_{1}\left(\left\{y \in X_{1}: f_{1}(y) \geq s\right\}\right)+\mu_{2}\left(\left\{z \in X_{2}: f_{2}(z) \geq 1-s\right\}\right)\right) .\right.
\end{aligned}
$$

Hence, for every $f_{i} \in \mathcal{L}\left(X_{i}\right)$ such that $\chi_{F} \leq \sum_{i=1}^{2} f_{i} \circ \pi_{i}$,

$$
\sum_{i=1}^{2} \int f_{i} d \mu_{i} \geq \bigwedge_{0 \leq s \leq 1}\left(\mu_{1}\left(\left\{y \in X_{1}: f_{1}(y) \geq s\right\}\right)+\mu_{2}\left(\left\{z \in X_{2}: f_{2}(z) \geq 1-s\right\}\right)\right) .
$$


Taking into account that

$$
I(F)=\bigwedge\left\{\sum_{i=1}^{2} \int \chi_{B_{i}} d \mu_{i}: B_{i} \in \mathcal{B}\left(X_{i}\right) \text { and } \chi_{F} \leq \sum_{i=1}^{2} \chi_{B_{i}} \circ \pi_{i}\right\}
$$

since for every $f_{i} \in \mathcal{L}\left(X_{i}\right)$, for every $t \in[0,1]$, the set $\left\{x \in X_{i}: f_{i}(x) \geq t\right\}$ is a Borel subset of $X_{i}$, it follows that

$$
\begin{aligned}
I(F) \geq & \bigwedge\left\{\sum_{i=1}^{2} \int f_{i} d \mu_{i}: f_{i} \in \mathcal{L}\left(X_{i}\right) \text { and } \chi_{F} \leq \sum_{i=1}^{2} f_{i} \circ \pi_{i}\right\} \\
\geq & \bigwedge\left\{\bigwedge_{0 \leq s \leq 1}\left(\mu_{1}\left(\left\{x \in X_{1}: f_{1}(x) \geq s\right\}\right)+\mu_{2}\left(\left\{z \in X_{2}: f_{2}(z) \geq 1-s\right\}\right)\right):\right. \\
& \left.f_{i} \in \mathcal{L}\left(X_{i}\right) \text { and } \chi_{F} \leq \sum_{i=1}^{2} f_{i} \circ \pi_{i}\right\} \\
\geq & I(F) .
\end{aligned}
$$

So $(*)$ holds.

2. In this step we prove that, for every closed set $C \in \mathcal{B}\left(X_{1} \times X_{2}\right)$,

$$
\text { (**) } \begin{aligned}
& \bigwedge\left\{\sum_{i=1}^{2} \int f_{i} d \mu_{i}: f_{i} \in \mathcal{L}\left(X_{i}\right) \text { and } \chi_{C} \leq \sum_{i=1}^{2} f_{i} \circ \pi_{i}\right\} \\
= & \bigwedge\left\{\sum_{i=1}^{2} \int h_{i} d \mu_{i}: h_{i} \in \mathcal{U}\left(X_{i}\right) \text { and } \chi_{C} \leq \sum_{i=1}^{2} h_{i} \circ \pi_{i}\right\}
\end{aligned}
$$

where $\mathcal{U}\left(X_{i}\right)$ is the family of all upper semicontinuous elements of $\mathcal{L}\left(X_{i}\right)$. Let $x^{*}$ be a nonnegative element of $\mathbf{B}^{*}$. By Proposition 1.31 of [9], for the real valued tight measures $x^{*} \circ \mu_{1}$ and $x^{*} \circ \mu_{2}$, the following equality holds

$$
\begin{aligned}
& \inf \left\{\sum_{i=1}^{2} \int f_{i} d\left(x^{*} \circ \mu_{i}\right): f_{i} \in \mathcal{L}\left(X_{i}\right) \text { and } \chi_{C} \leq \sum_{i=1}^{2} f_{i} \circ \pi_{i}\right\} \\
= & \inf \left\{\sum_{i=1}^{2} \int h_{i} d\left(x^{*} \circ \mu_{i}\right): h_{i} \in \mathcal{U}\left(X_{i}\right) \text { and } \chi_{C} \leq \sum_{i=1}^{2} h_{i} \circ \pi_{i}\right\} .
\end{aligned}
$$


Hence, for every nonnegative element $x^{*}$ of $\mathbf{B}^{*}$ and for every $f_{i} \in \mathcal{L}\left(X_{i}\right)$ such that $\chi_{C} \leq \sum_{i=1}^{2} f_{i} \circ \pi_{i}$,

$$
\begin{aligned}
& x^{*}\left(\sum_{i=1}^{2} \int f_{i} d \mu_{i}\right)=\sum_{i=1}^{2} \int f_{i} d\left(x^{*} \circ \mu_{i}\right) \\
& \geq \inf \left\{\sum_{i=1}^{2} \int h_{i} d\left(x^{*} \circ \mu_{i}\right): h_{i} \in \mathcal{U}\left(X_{i}\right) \text { and } \chi_{C} \leq \sum_{i=1}^{2} h_{i} \circ \pi_{i}\right\} \\
& =\inf \left\{x^{*}\left(\sum_{i=1}^{2} \int h_{i} d \mu_{i}\right): h_{i} \in \mathcal{U}\left(X_{i}\right) \text { and } \chi_{C} \leq \sum_{i=1}^{2} h_{i} \circ \pi_{i}\right\} \\
& \geq x^{*}\left(\bigwedge\left\{\sum_{i=1}^{2} \int h_{i} d \mu_{i}: h_{i} \in \mathcal{U}\left(X_{i}\right) \text { and } \chi_{C} \leq \sum_{i=1}^{2} h_{i} \circ \pi_{i}\right\}\right) .
\end{aligned}
$$

Therefore, for every $f_{i} \in \mathcal{L}\left(X_{i}\right)$ such that $\chi_{C} \leq \sum_{i=1}^{2} f_{i} \circ \pi_{i}$,

$$
\sum_{i=1}^{2} \int f_{i} d \mu_{i} \geq \bigwedge\left\{\sum_{i=1}^{2} \int h_{i} d \mu_{i}: h_{i} \in \mathcal{U}\left(X_{i}\right) \text { and } \chi_{C} \leq \sum_{i=1}^{2} h_{i} \circ \pi_{i}\right\} .
$$

So we obtain that

$$
\begin{aligned}
& \bigwedge\left\{\sum_{i=1}^{2} \int f_{i} d \mu_{i}: f_{i} \in \mathcal{L}\left(X_{i}\right) \text { and } \chi_{C} \leq \sum_{i=1}^{2} f_{i} \circ \pi_{i}\right\} \\
\geq & \bigwedge\left\{\sum_{i=1}^{2} \int h_{i} d \mu_{i}: h_{i} \in \mathcal{U}\left(X_{i}\right) \text { and } \chi_{C} \leq \sum_{i=1}^{2} h_{i} \circ \pi_{i}\right\} \\
\geq & \bigwedge\left\{\sum_{i=1}^{2} \int f_{i} d \mu_{i}: f_{i} \in \mathcal{L}\left(X_{i}\right) \text { and } \chi_{C} \leq \sum_{i=1}^{2} f_{i} \circ \pi_{i}\right\} .
\end{aligned}
$$

3. Now we show that, for every closed set $C \in \mathcal{B}\left(X_{1} \times X_{2}\right)$,

$$
\begin{aligned}
(* * *) & \bigwedge\left\{\sum_{i=1}^{2} \mu_{i}\left(C_{i}\right): C_{i} \text { closed set in } \mathcal{B}\left(X_{i}\right) \text { and } C \subseteq \cup_{i=1}^{2} \pi_{i}^{-1}\left(C_{i}\right)\right\} \\
= & \bigwedge\left\{\sum_{i=1}^{2} \int h_{i} d \mu_{i}: h_{i} \in \mathcal{U}\left(X_{i}\right) \text { and } \chi_{C} \leq \sum_{i=1}^{2} h_{i} \circ \pi_{i}\right\} .
\end{aligned}
$$


We only have to notice that, for every $h_{i} \in \mathcal{U}\left(X_{i}\right)$, for every $t \in[0,1]$, the set $\left\{x \in X_{i}: h_{i}(x) \geq t\right\}$ is a closed subset of $X_{i}$, and to argue as in step 1.

4. Conclusion. By the previous steps,

$$
\begin{aligned}
I(C) & =\bigwedge\left\{\sum_{i=1}^{2} \int f_{i} d \mu_{i}: f_{i} \in \mathcal{L}\left(X_{i}\right) \text { and } \chi_{C} \leq \sum_{i=1}^{2} f_{i} \circ \pi_{i}\right\} \\
& =\bigwedge\left\{\sum_{i=1}^{2} \int h_{i} d \mu_{i}: h_{i} \in \mathcal{U}\left(X_{i}\right) \text { and } \chi_{C} \leq \sum_{i=1}^{2} h_{i} \circ \pi_{i}\right\} \\
& =\bigwedge\left\{\sum_{i=1}^{2} \mu_{i}\left(C_{i}\right): C_{i} \text { closed set in } \mathcal{B}\left(X_{i}\right) \text { and } C \subseteq \cup_{i=1}^{2} \pi_{i}^{-1}\left(C_{i}\right)\right\}
\end{aligned}
$$

So the theorem is proved.

Proposition 3.3. For each $F \in \mathcal{P}\left(X_{1} \times X_{2}\right), S(F) \leq I(F)$.

Proof. For any $F \in \mathcal{P}\left(X_{1} \times X_{2}\right), 0 \leq I(F)$, therefore the case $M=\emptyset$ is obvious. Otherwise it is enough to notice that, for every $\mu$ with marginals $\mu_{1}$ and $\mu_{2}$ and for every $\left(B_{1}, B_{2}\right) \in \mathcal{B}\left(X_{1}\right) \times \mathcal{B}\left(X_{2}\right)$ with $F \subseteq \cup_{i=1}^{2} \pi_{i}{ }^{-1}\left(B_{i}\right)$,

$$
\begin{aligned}
\mu^{*}(F) & \leq \mu^{*}\left(\cup_{i=1}^{2} \pi_{i}^{-1}\left(B_{i}\right)\right)=\mu\left(\cup_{i=1}^{2} \pi_{i}^{-1}\left(B_{i}\right)\right) \\
& \leq \sum_{i=1}^{2} \mu\left(\pi_{i}{ }^{-1}\left(B_{i}\right)\right)=\sum_{i=1}^{2} \mu_{i}\left(B_{i}\right) .
\end{aligned}
$$

Definition 3.4. [4, Definition 14, page 7] Let $\mathcal{F}$ be a field of subsets of a set $X$ and let $\mu: \mathcal{F} \rightarrow \mathbf{B}$ be a vector measure with values in a Banach space $\mathbf{B}$. The vector measure $\mu$ is said to be strongly additive whenever given a sequence $\left(F_{n}\right)_{n \in N}$ of pairwise disjoint members of $\mathcal{F}$, the series $\sum_{n=1}^{\infty} \mu\left(F_{n}\right)$ converges in norm.

Theorem 3.5. Let $\mathcal{F}$ be a field of subsets of a set $X$. Then every vector measure $\mu: \mathcal{F} \rightarrow \mathbf{B}^{+}$taking values in the positive cone of a Banach lattice with order continuous norm is strongly additive.

Proof. If $\left(F_{n}\right)_{n \in N}$ is a sequence of pairwise disjoint sets in $\mathcal{F}$, then the sequence of partial sums $s_{n}=\sum_{i=1}^{n} \mu\left(F_{i}\right)$ is increasing and it is bounded above by $\mu(X)$, hence it is directed $(\leq)$ and majorized. Thus, by [11, Theorem 5.10], the infinite series $\sum_{n=1}^{\infty} \mu\left(F_{n}\right)$ converges weakly. Therefore, by [11, Corollary, page 89], it norm converges. 
Definition 3.6. Let $\mathcal{A}$ and $\mathcal{B}$ be fields of subsets of a set $X_{1}$ and a set $X_{2}$, respectively. Henceforward by $\mathcal{A} \times \mathcal{B}$ we denote the field on $X_{1} \times X_{2}$ generated by all rectangles $A \times B$ for $A \in \mathcal{A}$ and $B \in \mathcal{B}$. By $\mathcal{A} \otimes \mathcal{B}$ we denote the $\sigma$-field on $X_{1} \times X_{2}$ generated by $\mathcal{A} \times \mathcal{B}$.

Given a non-empty set $X$ and a subset $F$ of $X$ by $F^{c}$ we denote the complement $X-F$ of $F$.

Theorem 3.7. Let $\mathcal{A}$ and $\mathcal{B}$ be fields of subsets of a set $X_{1}$ and a set $X_{2}$, respectively, and let $\mu_{1}: \mathcal{A} \rightarrow \mathbf{B}^{+}$and $\mu_{2}: \mathcal{B} \rightarrow \mathbf{B}^{+}$be vector measures taking values in the positive cone of an order complete Banach lattice $\mathbf{B}$. We assume that $\mu_{1}\left(X_{1}\right)=\mu_{2}\left(X_{2}\right)=\alpha$, for some $\alpha \in \mathbf{B}^{+}$. Let $F$ be an arbitrary set in $\mathcal{A} \times \mathcal{B}$ and let $\mathcal{C}$ be the field on $X_{1} \times X_{2}$ generated by $F$ and the sets in $\mathcal{A} \times \mathcal{B}$. For an element $v \in \mathbf{B}$, with $0 \leq v \leq \alpha$, we consider the following conditions:

(i) There is a vector measure $\mu: \mathcal{C} \rightarrow \mathbf{B}^{+}$such that $\mu\left(A \times X_{2}\right)=\mu_{1}(A)$ and $\mu\left(X_{1} \times B\right)=\mu_{2}(B)$ for all $A \in \mathcal{A}$ and $B \in \mathcal{B}$ (i.e. $\mu$ has marginals $\mu_{1}$ and $\left.\mu_{2}\right)$ and such that $\mu(F)=v$.

(ii) Whenever $A \times B \subseteq F$ for $A \in \mathcal{A}$ and $B \in \mathcal{B}$, then $\mu_{1}(A)+\mu_{2}(B) \leq \alpha+v$.

(iii) Whenever $A \times B \subseteq F^{c}$ for $A \in \mathcal{A}$ and $B \in \mathcal{B}$, then $\mu_{1}(A)+\mu_{2}(B) \leq$ $2 \alpha-v$.

Then (i) is equivalent to the conjunction of (ii) and (iii).

Proof. This follows from Theorem 2.1 in [7].

Theorem 3.8. Let $\mathcal{A}$ and $\mathcal{B}$ be $\sigma$-fields of subsets of a set $X_{1}$ and a set $X_{2}$, respectively, and let $\mu: \mathcal{A} \times \mathcal{B}: \rightarrow \mathbf{B}^{+}$be a vector measure, where $\mathbf{B}^{+}$is the positive cone of a Banach lattice $\mathbf{B}$. Define $\mu_{1}: \mathcal{A} \rightarrow \mathbf{B}^{+}$and $\mu_{2}: \mathcal{B} \rightarrow \mathbf{B}^{+}$ by the rule $\mu_{1}(A)=\mu\left(A \times X_{2}\right)$ and $\mu_{2}(B)=\mu\left(X_{1} \times B\right)$.

If $\mu_{1}$ is compact and $\mu_{2}$ is countably additive, then $\mu$ is countably additive on $\mathcal{A} \times \mathcal{B}$.

Proof. This result is proved in the first part of the proof of Theorem 3.1 of [12].

Theorem 3.9. Let $\mathcal{A}$ and $\mathcal{B}$ be $\sigma$-fields of subsets of a set $X_{1}$ and a set $X_{2}$, respectively, and let $\mu_{1} \in c a\left(\mathcal{A}, \mathbf{B}^{+}\right)$and $\mu_{2} \in c a\left(\mathcal{B}, \mathbf{B}^{+}\right)$, with $\mu_{1}\left(X_{1}\right)=$ $\mu_{2}\left(X_{2}\right)=\alpha$, where $\mathbf{B}^{+}$is the positive cone of a Banach lattice $\mathbf{B}$ with order continuous norm. Suppose that $\mu_{1}$ is compact and that $F \in \mathcal{A} \otimes \mathcal{B}$ is a countable intersection of sets in $\mathcal{A} \times \mathcal{B}$. For any $v \in \mathbf{B}^{+}$, with $v \leq \alpha$, the following are equivalent: 
(i) There is a vector measure $\mu \in c a\left(\mathcal{A} \otimes \mathcal{B}, \mathbf{B}^{+}\right)$with $\mu \circ \pi_{1}{ }^{-1}=\mu_{1}$ and $\mu \circ \pi_{2}^{-1}=\mu_{2}$ such that $\mu(F) \geq v$.

(ii) For all $A \in \mathcal{A}$ and $B \in \mathcal{B}$, we have $\mu_{1}(A)+\mu_{2}(B) \leq 2 \alpha-v$, whenever $A \times B \subseteq F^{c}$.

Proof. $(i) \Rightarrow(i i)$ Since

$$
\begin{aligned}
& A \times B \subseteq F^{c} \Rightarrow F \subseteq\left(A^{c} \times X_{2}\right) \cup\left(X_{1} \times B^{c}\right) \\
\Rightarrow & \left(A \times X_{2}\right) \cap F \subseteq\left(A \times X_{2}\right) \cap\left(\left(A^{c} \times X_{2}\right) \cup\left(X_{1} \times B^{c}\right)\right) \\
\Rightarrow & \left(A \times X_{2}\right) \cap F \subseteq A \times B^{c},
\end{aligned}
$$

we calculate

$$
\begin{aligned}
& \mu_{1}(A)=\mu\left(A \times X_{2}\right)=\mu\left(\left(A \times X_{2}\right) \cap F\right)+\mu\left(\left(A \times X_{2}\right) \cap F^{c}\right) \\
\leq & \mu\left(X_{1} \times B^{c}\right)+\mu\left(\left(X_{1} \times X_{2}\right)-F\right)=\mu_{2}\left(B^{c}\right)+\mu\left(X_{1} \times X_{2}\right)-\mu(F) \\
\leq & 2 \alpha-\mu_{2}(B)-v .
\end{aligned}
$$

$($ ii $) \Rightarrow($ i $)$ As in the proof of Theorem 2 in [8], define $I=\bigwedge\left\{2 \alpha-\mu_{1}(A)-\right.$ $\left.\mu_{2}(B): A \times B \subseteq F^{c}\right\}$ and $\Sigma=\bigvee\left\{\mu_{1}(A)+\mu_{2}(B)-\alpha: A \times B \subseteq F\right\}$. It is straightforward that $\Sigma \leq I$ : suppose that $A \times B \subseteq F$ and $A_{0} \times B_{0} \subseteq F^{c}$. Note that either $A \cap A_{0}=\emptyset$ or $B \cap B_{0}=\emptyset$. Therefore, $\mu_{1}(A)+\mu_{2}(B)+$ $\mu_{1}\left(A_{0}\right)+\mu_{2}\left(B_{0}\right) \leq 3 \alpha$, and hence $\mu_{1}(A)+\mu_{2}(B)-\alpha \leq 2 \alpha-\mu_{1}\left(A_{0}\right)-\mu_{2}\left(B_{0}\right)$, as desired.

Let $v_{0}=v \vee \Sigma$. Since, by (ii), whenever $A \times B \subseteq F^{c}$, we have $v \leq$ $2 \alpha-\mu_{1}(A)-\mu_{2}(B)$, it is clear that $\Sigma \leq v_{0} \leq I$. Hence, (ii) and (iii) of Theorem 3.7 hold with $v_{0}$ in place of $v$. Let $\mathcal{C}$ be the field generated by $\mathcal{A} \times \mathcal{B}$ and the set $F$. By Theorem 3.7, there exists a vector measure $\mu_{0}: \mathcal{C} \rightarrow \mathbf{B}^{+}$ with marginals $\mu_{1}$ and $\mu_{2}$ and such that $\mu_{0}(F)=v_{0}$. By Theorem $3.8 \mu_{0}$ is countably additive on $\mathcal{A} \times \mathcal{B}$, because it has countably additive marginals, one of which is compact. Using Theorem 3.5 and Kluvanek's Theorem [4, page 27], we find a countably additive vector measure $\mu: \mathcal{A} \otimes \mathcal{B} \rightarrow \mathbf{B}^{+}$such that $\mu=\mu_{0}$ on $\mathcal{A} \times \mathcal{B}$. Choose a decreasing sequence of sets $\left(F_{n}\right)_{n \in N}$ in $\mathcal{A} \times \mathcal{B}$ such that $\cap_{n \in N} F_{n}=F$. Then

$$
\mu(F)=\lim _{n} \mu\left(F_{n}\right)=\lim _{n} \mu_{0}\left(F_{n}\right) \geq \mu_{0}(F)=v_{0} \geq v,
$$

establishing the theorem. 
Theorem 3.10. Let $\mathcal{A}$ and $\mathcal{B}$ be $\sigma$-fields of subsets of a set $X_{1}$ and a set $X_{2}$, respectively, and let $\mu_{1} \in c a\left(\mathcal{A}, \mathbf{B}^{+}\right)$and $\mu_{2} \in c a\left(\mathcal{B}, \mathbf{B}^{+}\right)$, with $\mu_{1}\left(X_{1}\right)=$ $\mu_{2}\left(X_{2}\right)=\alpha$, where $\mathbf{B}^{+}$is the positive cone of a Banach lattice $\mathbf{B}$ with order continuous norm. Suppose that $\mu_{1}$ is perfect and that $F \in \mathcal{A} \otimes \mathcal{B}$ is a countable intersection of sets in $\mathcal{A} \times \mathcal{B}$. For any $v \in \mathbf{B}^{+}$, with $v \leq \alpha$, the following are equivalent:

(i) There is a vector measure $\mu \in c a\left(\mathcal{A} \otimes \mathcal{B}, \mathbf{B}^{+}\right)$with $\mu \circ \pi_{1}^{-1}=\mu_{1}$ and $\mu \circ \pi_{2}{ }^{-1}=\mu_{2}$ such that $\mu(F) \geq v$.

(ii) For all $A \in \mathcal{A}$ and $B \in \mathcal{B}$, we have $\mu_{1}(A)+\mu_{2}(B) \leq 2 \alpha-v$, whenever $A \times B \subseteq F^{c}$.

Proof. The proof is the same as the one of Theorem 3.9. The only difference is the fact that instead of applying Theorem 3.8 we apply Theorem 3.1 of [12].

Proposition 3.11. [2, Proposition 8.1.5] Let $X_{1} \ldots X_{n} \ldots$ be a (finite or infinite) sequence of separable metrizable spaces. Then $\mathcal{B}\left(\Pi_{i} X_{i}\right)=\otimes_{i} \mathcal{B}\left(X_{i}\right)$.

Theorem 3.12. Let $X_{1}$ and $X_{2}$ be Polish spaces and let $\mu_{1}$ and $\mu_{2}$ be elements of $c a\left(\mathcal{B}\left(X_{1}\right), \mathbf{B}^{+}\right)$and of $c a\left(\mathcal{B}\left(X_{2}\right), \mathbf{B}^{+}\right)$, respectively, with $\mu_{1}\left(X_{1}\right)=\mu_{2}\left(X_{2}\right)=$ $\alpha$, where $\mathbf{B}^{+}$is the positive cone of a Banach lattice $\mathbf{B}$ with order continuous norm. Let $F \in \mathcal{B}\left(X_{1} \times X_{2}\right)$ be a countable intersection of sets in $\mathcal{B}\left(X_{1}\right) \times$ $\mathcal{B}\left(X_{2}\right)$. For any $v \in \mathbf{B}^{+}, v \leq \alpha$, the following are equivalent:

(1) There is a vector measure $\mu$ in $\operatorname{ca}\left(\mathcal{B}\left(X_{1} \times X_{2}\right)\right.$, $\left.\mathbf{B}^{+}\right)$with $\mu \circ \pi_{1}^{-1}=\mu_{1}$ and $\mu \circ \pi_{2}^{-1}=\mu_{2}$ such that $\mu(F) \geq v$.

(2) $I(F) \geq v$.

Proof. It is enough to observe that $\mu_{1}$ and $\mu_{2}$ are tight [10, Theorem 3.2] and that hypothesis (2) is equivalent to (ii) of Theorem 3.9.

Corollary 3.13. Let $X_{1}$ and $X_{2}$ be Polish spaces and let $\mu_{1}$ and $\mu_{2}$ be elements of $c a\left(\mathcal{B}\left(X_{1}\right), \mathbf{B}^{+}\right)$and of $c a\left(\mathcal{B}\left(X_{2}\right), \mathbf{B}^{+}\right)$, respectively, with $\mu_{1}\left(X_{1}\right)=\mu_{2}\left(X_{2}\right)=$ $\alpha$, where $\mathbf{B}^{+}$is the positive cone of a Banach lattice $\mathbf{B}$ with order continuous norm. Then there exists $\mu$ in $c a\left(\mathcal{B}\left(X_{1} \times X_{2}\right), \mathbf{B}^{+}\right)$with $\mu \circ \pi_{1}{ }^{-1}=\mu_{1}$ and $\mu \circ \pi_{2}^{-1}=\mu_{2}$ (i.e., under these hypotheses, the set $M$ of Definition 3.1 is non-empty).

Proof. We only have to notice that $I\left(X_{1} \times X_{2}\right)=\alpha$ and to apply Theorem 3.12 with $X_{1} \times X_{2}$ in place of $F$ and with $v=\alpha$. 
Theorem 3.14. Duality Theorem. Let $X_{1}$ and $X_{2}$ be Polish spaces and let $\mu_{1} \in c a\left(\mathcal{B}\left(X_{1}\right), \mathbf{B}^{+}\right)$and $\mu_{2} \in c a\left(\mathcal{B}\left(X_{2}\right), \mathbf{B}^{+}\right)$, with $\mu_{1}\left(X_{1}\right)=\mu_{2}\left(X_{2}\right)=\alpha$, where $\mathbf{B}^{+}$is the positive cone of a Banach lattice $\mathbf{B}$ with order continuous norm. Let $F \in \mathcal{B}\left(X_{1} \times X_{2}\right)$ be a countable intersection of sets in $\mathcal{B}\left(X_{1}\right) \times$ $\mathcal{B}\left(X_{2}\right)$. Then

$$
I(F)=S(F) .
$$

Proof. Let $v=I(F)$. By Theorem 3.12, there exists $\mu$ in ca $\left(\mathcal{B}\left(X_{1} \times X_{2}\right), \mathbf{B}^{+}\right)$ with $\mu \circ \pi_{1}^{-1}=\mu_{1}, \mu \circ \pi_{2}^{-1}=\mu_{2}$ and $\mu(F) \geq v$. Thus, $S(F) \geq I(F)$. This inequality and Proposition 3.3 lead to the desired equality.

The next result extends Proposition 3.8 of [9] to the vector case.

Theorem 3.15. Let $X_{1}$ and $X_{2}$ be Polish spaces and let $\mu_{1}$ and $\mu_{2}$ be elements of $\operatorname{ca}\left(\mathcal{B}\left(X_{1}\right), \mathbf{B}^{+}\right)$and of $c a\left(\mathcal{B}\left(X_{2}\right), \mathbf{B}^{+}\right)$, respectively, with $\mu_{1}\left(X_{1}\right)=\mu_{2}\left(X_{2}\right)=$ $\alpha$, where $\mathbf{B}^{+}$is the positive cone of a Banach lattice $\mathbf{B}$ with order continuous norm. Let $C$ be a closed subset of $X_{1} \times X_{2}$. Then, for any $v \in \mathbf{B}^{+}, v \leq \alpha$, there exists a vector measure $\mu \in c a\left(\mathcal{B}\left(X_{1} \times X_{2}\right), \mathbf{B}^{+}\right)$such that $\mu \circ \pi_{1}{ }^{-1}=\mu_{1}$ and $\mu \circ \pi_{2}^{-1}=\mu_{2}$, with $\mu(C) \geq v$ if and only if

$$
\sum_{i=1}^{2} \mu_{i}\left(C_{i}\right) \geq v, \text { for all } C_{i} \text { closed sets in } \mathcal{B}\left(X_{i}\right) \text { with } C \subseteq \cup_{i=1}^{2} \pi_{i}{ }^{-1}\left(C_{i}\right) \text {. }
$$

Proof. Since $C=\cap_{j \in J}\left(A_{1, j} \times A_{2, j}\right)^{c}$, with $A_{i, j}$ open subset of $X_{i}, J$ finite or countable, $i=1,2$, this follows at once from Theorem 3.2 and Theorem 3.12 .

\section{References}

[1] C. D. Aliprantis and O. Burkinshaw, Positive Operators, Pure and Applied Mathematics Series, Academic Press, New York and London, 1985.

[2] D. I. Cohn, Measure Theory, Birkhäuser, Boston, 1980.

[3] E. D'Aniello and R. M. Shortt, Vector-valued Capacities, Rendiconti del Circolo Matematico di Palermo, Serie II, vol. XLVII (1998), 311-324.

[4] J. Diestel and J. J. Uhl, Vector Measures, Amer. Math. Soc., Mathematical Surveys and Monographs N. 15, Providence (RI), 1977.

[5] R. M. Dudley, Real Analysis and Probability, Chapman and Hall Mathematics Series, New York and London, 1989. 
[6] D. A. Edwards, On the Existence of Probability Measures with Given Marginals, Ann. Inst. Fourier 28/4(1978), 53-78.

[7] A. Hirshberg and R. M. Shortt, Strassen's Theorem for group-valued charges, Distributions with given Marginals and Moment Problems, V. Beneš and J. Štěpán, eds., Kluwer Academic Publishers, Dordrecht Boston - London (1997), 173-178.

[8] A. Hirshberg and R. M. Shortt, A Version of Strassen's Theorem for Vector-valued Measures, Proceedings of the Amer. Math. Soc. 126(1998), 1669-1671.

[9] H. G. Kellerer, Duality Theorems for Marginal Problems, Z. Wahrscheinlichkeitstheor. Verw. Geb. 67(1984), 399-432.

[10] M. März and R. M. Shortt, Weak Convergence of Vector Measures, Publ. Math. (Debrecen) vol. 45(1994), 71-92.

[11] H. H. Schaefer, Banach Lattices and Positive Operators, Springer-VerlagBerlin, 1974.

[12] R. M. Shortt, Strassen's Theorem for vector measures, Proc. Amer. Math. Soc. 1226 (1994), 811-820.

[13] V. Strassen, The Existence of Probability Measures with Given Marginals, Ann. Math. Statist. vol. 36 (1965), 423-439. 